\title{
Dic Funktion von Bildern und Motiven zu Ägypten und Kairo in der deutschsprachigen Literatur der Gegenwart
}

Ägypten in der Sicht der Weltliteratur" - so das Thema dieses Symposiums, und diese Formulierung läßt großen Spielraum. Ägypten als Motiv, die literarischen, also sprachlichen Bilder Ägyptens - diese möglichst am konkreten Beispiel, am literarischen Text zu ergründen und zu beschreiben, macht eine grundlegende, vorgängige Entscheidung notwendig. Will man den Spuren eines realen Ägypten, seiner Geschichte, seiner Kultur, seiner Mythen etwa in ihrer sprachlichliterarischen Abgebildetheit nachforschen, so braucht man den methodischen, und damit begrenzenden Zugriff, beschränkt sich etwa auf eine Epoche, auf ein spezifisches Motiv, auf einen Autor usw. (Einige Beiträge dazu waren auf diesem Symposium zu hören.) Eine andere Art der Annäherung geht den ungekelirten Weg, indem sie von einem Begriff von Literatur ausgeht, der behauptet, in der Literatur geht es, wenn es um Ägypten geht, zumeist nicht um Ägypten - und versucht dann zu ergründen, inwiefern und warım Ägypten für etwas anderes steht, und in welchen Zusammenhang mit diesem Anderen es steht. Beide Vorgehensweisen sind begründet und legitim, beide können einander ergänzen, selbst dann, wenn sie auf denselben Text angewandt werden.

Ich möchte anhand einiger kurzer Bemerkungen zu zwei neueren Texten ${ }^{1}$, die beide im Zeitraum der letzten 20 Jahre entstanden sind, zu zeigen versuchen, daß die literarische Abbildung Ägyptens, die ja immer nur in kleinsten Ausschnitten möglich ist, eine parabolische ist - also Ägypten sagt, aber etwas anderes meint. Ich gehe dabei von der These aus, daß der Ägypten-Stoff in der Literatur schon immer ein literarisches Ägypten zur

\footnotetext{
${ }^{1}$ In einem ähnlichen Zusammenhang habe ich die beiden hier behandelten Romane zusammen mit weiteren literarischen Texten aus den letzten Jahren in einem frülıer veröffentlichten Aufsat'z untersucht: Das Selbst am fremden Ort. Kairo als Raum für Geschichte in Texten deatschsprachiger Autoren der Gegenwart. Mit cinem Ausblick auf einen Roman der ägyptischen Autorin Alıdaf Soueiᄃ, in: Jahrbuch Deutsch als Frcmdsprachc. Intcrcultural Gcrman Studics, Bd. 28, 2002, S. $39-60$.
} 
Grundlage hatte. Mit anderen Worten: Ägypten in der Literatur ist nicht das Abbild eines realen, sondern eines schon zuvor literarisierten Ägyptens, also das Abbild eines Konstruktes in Worten.

Das literarische Ägypten ist ein mentales Gebilde, in dem eigene - d.h. für dieses Symposium: europäische - Rezeptionsweisen tradiert sind, in dem Mythos und Historie, gegenwärtige Vorurteile und stereotype Bilder zu einer Substanz verdichtet sind, die dem Autor als Ausgangsmaterial für seine literarischen Verfremdungen dienen. Womit ein weiterer Vorgang angesprochen ist, der der Literatur immer schon eigen ist: der des Verfremdens gewohnter Perzeptionsweisen. Das Verfremden von Ereignissen, Personen und Orten in der Literatur zielt dabei auf das dem Leser Bekannte, dessen Wahmelmung entautomatisiert werden soll, un neue Sehweisen zu schaffen. Handelt es sich bei dem Objekt der literarischen Darstellung aber um etwas, das nicht dem Erfalınungsraum des Lesers entstammt, ihm, wenn nicht unbekannt, so doch erfahrungsfremd ist - wie z.B. der kulturell anders geprägte Ort - wird auch dieses wiederum verfremdet. Versteht man unter "Verfremdung" das „Fremdmachen“" von Bekanntem, so liegt auf der Hand, daß mit der literarischen Darstellung von Ägypten im weitesten Sinne nicht das real erfahrbare Ägypten, sondern - in unserem Zusammenhang: europäische Vorstellungen und mentale Konzepte von diesem Land und seiner Kultur verfremdet werden. Geht man weiterhin davon aus, daß Literatur kommunikativ ist, indem sie nicht einfach Informationen und Erkenntnisse, sondem deren Verarbeitung vermittelt - also eine mehr oder weniger konkret verortbare Ereigniskette in ihren Verstehensmöglichkeiten offeriert - so muß auch der Ort der Ereignisse selbst ein zu verstehender sein, jenseits seiner real zu erfalırenden Erscheinung. Und dieses Verstehen, das Literatur zugleich anbietet und vom Leser fordert, ist kulturgebunden.

So weit die hier nur knapp angesprochenen Prämissen. Was heißt dies nun, konkret am Beispiel der Darstellung Ägyptens in jüngsten deutschsprachigen literarischen Texten? Wenn Ägypten nicht Ägypten ist, dann ist es doch immer das, was man damit nachvollziehbar verbinden kann. Die Pyramiden z.B. stehen für das, was diese Bauwerke den Menschen schon immer auch bedeutet haben, also für ein Mehr ihrer reinen Anschaulichkeit. Ein Beispiel:

Ich hatte viel von ihr gehört, viel über sie gelesen, und ilur Erscheinungsbild war mir so gut vertraut wie ilıre Herkunft und ihre Körpermaße, und alles, was ich wußte und mir während der Fahrt zu ihr noch einmal nicht olne Erregung 
vergegenwärtigte, deutete Großes an. Trotzdem verlor ich, als ich vor ihr stand, die Fassung, die Knie zitterten mir, ich schrumpfte. Nie zuvor hatte eine wirkliche Wirklichkeit mein Bild von ihr so entschieden verkläglicht, daß ich mich meiner Vorstellungskraft hätte schämen müssen. Nun tat ich es - wenn auch nur für ein paar Augenblicke -, denn vor dem sinnlichen Da des Wunders, ich spürte es, wird alle Malerei des Hirns zum bleichen Pfusch. ${ }^{2}$

So beginnt der Roman von Markus Werner, „Der ägyptische Heinrich“. Der Erzähler steht vor der gewaltigsten der Pyramiden, der CheopsPyramide, deren mentales Bild er aus der schweizerischen Heimat mitgebracht hat. Nichts Fremdem also begegnet der Europäer hier im Angesicht des ägyptischen Weltwunders, sondern etwas Wohlbekanntem, einer in seiner Kultur ständig vermittelten Größe, deren Erscheinung, ihre "wirkliche Wirklichkeit“, ihn allerdings zunächst überwältigt und ihn erfahren läßt, daß alle mentalen Konstrukte - ,alle Malerei des Hirns" vor dem sinnlichen Erlebnis verblassen. Die freudige Erwartung des bevorstehenden Erlebnisses, in der sprachlichen Formulierung auf ein erotisches Abenteuer hindeutend, entspringt einem fest geprägten Vorverstehen, das der Erzähler aus dem fernen Europa mitbringt.

An dieser Textstelle wird deutlich, daß es eine Vermittlung zwischen Sinnlich-Wahrgenommenem und kulturell Tradiertem erst dann geben kann, wenn die mentale Bearbeitung beider Erfalınngsweisen, ausgelōst durch wechselseitige Enttäuschung, beginnt. Die kurz darauf folgende Textstelle erhellt diesen Zusammenhang:

Ich stand in der Königskammer, ich stand in der Grabkammer, die in Wahrheit keine Kammer, sondern ein kleiner Saal ist, ein Grabsaal, leer bis auf den leeren Granitsarg des verschollenen Cheops.

Ich blieb nicht lange, es roch so arg nach Urin, daß ein gemessenes Verweilen nicht in Betracht kam. Auch huschte jetzt ein asiatisches Pärchen herein und sagte Good morning. Ich warf noch einen Blick in den offenen Sarkophag. Kaugummis, eine zerknüllte Tampaxschachtel, ich kroch zurück ans Licht. (ÄH 5 f.)

${ }^{2}$ Markus Wemer. „Der āgyptische Heinrich“, Salzburg/Wien (Residen7.), 1999. S. 5. In der Folge als $\bar{A} H$ abgekūrzt. 
Obwohl die Enttäuschung nicht explizit thematisiert wird, ist sie der lakonischen Beschreibung doch cindeutig zu cntnchmen. Der allzu weltliche und profane Geruch nach Urin, das globale und in diesem Sinne kulturindifferente „Good morning", zusammen mit alltäglichen und dem persönlich-intimen Bereich zugerechneten Utensilien entweihen und besudeln einen Ort, der in der europäischen Vorstellung ein geweihter Ort ist. Er ist ein Zeugnis für die hochwertige kulturelle Entwicklung der Menschlıcit, für ihre kulturellen Wurzelı, die über vielfältige Vermittlungen auch die empfundenen Wurzeln des Europäers sind. Damit ist die Spur gelegt, die die moderne Literatur zu einer Instanz der Aufklärung macht: die Suche nach den Wurzeln des Eigenen und des Selbst kommt an der nüchternen Analyse der Welt und ihrer Realitäten nicht vorbei. So ist auch das Zurückkriechen ,ans Licht" als ein der Enttäuschung entsprungener Akt im Sinne der aufklärerischen Vernunft zu verstehen: der Erzälıler verläßt das Dunkel der mythischen Herkunftsvnrstellungen und begibt sich ans Licht der Tatsachen.

Diese Deutung der Textstelle mag belegen, da $\beta$ auch das scheinbar Fremde in der Literatur nur dann Ausgangspunkt für Verstehen sein kann, wenn es als Fremdes schon vorab Bestandteil des Eigenen geworden ist. Die Pyramide ist fremd lediglich in ihrer sinnlichen Anschaulichkeit im Ersterlebnis des Erzählers, nicht aber in dem, für das sie gesehen wird, schon immer gesehen wurde. Das wäre, um nur einen Aspekt herauszugreifen aus der schier unendlichen Kette von Bezügen, die sich aus europäischer Perspektive im Bedeutungsfeld „Ägypten“ ergeben, zum Beispiel die Vorstellung von Ewigkeit, Dauer, dem Überdauern von Sinn, damit das Sehnsuchtsideal einer wie immer gearteten Unvergänglichkeit. Diese Unvergänglichkeit, die nur in den Religionen noch als ewiges Leben sinnhaft konstituiert ist - dort aber transzendiert in ein Leben nach dem Tode - will dem in der aufgeklärten europäischen Denkkultur verhafteten Erzähler in den Pyramiden aufscheinen. Da es nur dieses Leben für ihn gibt, werden die Pyramiden zur Kompensationsgröße seiner kulturellen Verlusterfahrung, die dem Empfinden der Entwurzelung und Unbeheimatetheit in der gegenwärtigen, schweizerischen Erfalırungswelt entspringt. Das Befremden durch die sinnliche, detaillierte und gegenwärtig-reale Anschaulichkeit wirft den Erzähler indessen zurück auf sein Vorverstehen, das enttäuscht wird, mit der Konsequenz einer neuen Erkenntnis, die das Befremdliche auslöst. Die verfremdende Darstellung der Pyramide korrespondiert in dem zitierten Textausschnitt überraschend mit einer äußerst realen: es ist gerade die gegenwärtige Erlebensrealität, die Erkenntnis des Tatsächlichen, die das in einem europäischen Verstehensrahmen Tradierte neu perspektiviert. Allzu bekannt erscheint das Fremde, fremd hingegen wird dem erlebenden 
Erzähler - und im Verarbeiten der literarischen Darstellung damit auch dem Leser - die eigene Vorstellungswelt, die bisher eine durch Schrift vermittelte war, und nun vor dem Erleben der „wirklichen Wirklichkeit“ zurückschreckt.

Ähnlich belegt eine Textstelle aus dem Roman „Wassermann“ von Silvio Blatter die Selınsuchtsprojektion auf Ägypten, die den Stillstand der Zeiten und damit ewige Sinnhaftigkeit beschwört:

Es geschieht nichts, es geschieht seit Jahrhunderten dasselbe. Frauen, die Last auf dem Kopf, Männer, die Last auf den Schultern, Tiere, die Last auf dem Rücken. Unter diesem blauen Himmel, der auch weh tut auf Rücken, Schultern und Kopf. Die Hitze zwingt einen, unangenehm bewußt zu atmen. (...) Verharrte die Feluka an Ort, zöge das Landleben vorbei, die Feldarbeit, es wäre wie ein Bilderbogen: Hassan Ali liest aus Gottfried Kellers Werken vor, so wäre das. ${ }^{3}$

Wiederum ist es die Schweiz, hier in der Bilderwelt eines ihrer bekanntesten Autoren, Gottfried Kellers, deren vergangene Lebenswelten auf einer Nilfahrt durch Oberägypten mit den Beobachtungen des ägyptischen Landlebens zur Deckung gebracht werden. Hier wird die sinnliche Anschaulichkeit eines fremden Landes zur Heimat umstilisiert: einer Heimat allerdings, die längst verloren ist und deshalb nur in einem Land wie Ägypten wiedergefunden werden kann. Was allerdings bedeutet, die eigene Verlusterfahrung auf Kosten der bewußten Erfahrung von Wirklichkeit zu kompensieren: das Verstehen gleichzeitiggegenwärtiger, fremder Lebensarten wird zugunsten eigener Herkunftsdeutung behindert, wenn nicht ausgeschlossen. Schmerzhafter Selbstverlust wird quasi therapeutisch geleugnet: die Reise mit der Feluka auf dem Nil ist eine Reise in die vermeintlich eigene Vergangenheit, in eine imaginierte Vorfahren- und Kindheitswelt, die den Verlust noch nicht kannte. Selbstversicherung also, bloßer Schein, dessen Entlarvung nicht lange auf sich warten läßt.

Nur das Erzählte kann als Ganzes noch erscheinen - dieser Bruch zwischen Erleben und Erzählen wird denn in den beiden Romanen auch immer wieder angesprochen. Sowohl Markus Werner als auch Silvio Blatter wenden sich in ihrem Erzählen, das vordergründig autobiographisch strukturiert ist, dieser Dualität auf zweifache Weise zu:

\footnotetext{
${ }^{3}$ Silvio Blatter: „Wassermann“, Frankfurt/M (Suhrkamp), 1986, S. 338. In der Folge als W abgekürt.
} 
einmal in der Reflexion der beiden Ich-Erzähler, die beide auf der Suche nach der eigenen Herkunft und Vergangenheit sind, die ihnen in der Imagination bruchlose Ganzheit suggeriert; zum anderen in der Anlage der Erzählstruktur, die in beiden Fällen eine zweigeteilte ist. Bei Markus Werner alterniert die Erzählung zwischen den Schauplätzen in der Schweiz - dort zum großen Teil auf Ereignisse im 19. Jahrhundert konzentriert, die den Lebensweg seines Urgroßvaters nachzeichnen - und dem gegenwärtigen Ägypten, in dem der Erzähler die Spuren des einst ausgewanderten Vorfahren sucht und die Stätten seines Wirkens aufsucht. Die Hoffnung richtet sich dabei allerdings auf ein Mehr: die Suche wird zur Reise in ein Verstehen des Andersseins, das dem Urgroßvater anhaftet, und das dem Erzähler so sympathisch ist, weil er selbst sich anders, ja entfremdet fühlt in einer Gegenwart, die ihm nicht mehr Heimat sein kann. Heimat wird für ihn zunehmend das die Gegenwart ausblendende, abgeschiedene Lesen in alten Schriften und biographischen Notizen des Vorfahren, in denen er auch aus Ägypten berichtet. Denn die Arbeit im Archiv in Zürich, zur Vorbereitung auf die Ägyptenreise, führt den Erzähler in eine Welt, die ihm weitaus vertrauter ist als die gegenwärtige Schweiz:

Manchmal, wenn ich aus einem der Archive Zürichs hinaustrat in die Gegenwart, bekam ich den bösen Blick. Ein einziger Tag der Versenkung schien bewirken zu können, $\mathrm{da} B$ ich mich beim Wiederauftauchen als Relikt jener Zeit fühlte, in der ich mich aufgehalten hatte, und die Rückkehr in meine Welt war keine ins Vertraute. (

Die Verlusterfahrung in Werners Roman ist eine doppelte. Denn auch die so heroisch erscheinende Biographie des Urgroßvaters stellt sich am Ende als so illusorisch heraus wie die Vorstellung, im Ägypten der Gegenwart sei all das zu finden, was man im Archiv überliefert vorfindet und als fehlendes Glied in einer lückenlos ersehnten Kette zu erkennen glaubt, die man wieder zu einem Ganzen zusammensetzen will. Das fehlende Glied symbolisiert dabei all das, was den mitteleuropäischen Kulturen verlorengegangen ist: Dauerhaftigkeit, Beständigkeit, Verläßlichkeit, Einfachheit, Besinnungsfähigkeit - und vor allem: Wahrhaftigkeit. Die Illusion liegt in der Vorstellung, der Inhalt dieser Begriffe sei in wirklich Erlebtem $\mathrm{zu}$ finden: etwa in der wirklich vorhandenen und $\mathrm{zu}$ besichtigenden Pyramide.

$\mathrm{Da}$ auch die reale ägyptische Pyramide nur in ihrer Bausubstanz überdauert, ist immerhin eine Erkenntnis, die die Verläßlichkeit des 
Erzählens wieder in den Vordergrund rückt: demn allein das Erzällen garantiert die Vereinigung von Teilerfahrungen in ein gemeinsames Ganzes, das über zeitliche wic kulturelle Grenzen hinweg den Verlust zumindest zeitweise als überwindbar erscheinen läßt. Im wahrsten Sinne des Wortes wird das Erzählen damit zu einem Zusammensetzen des in Einzelteile zerbrochenen Ganzen: Ägypten darin zum Ort, dessen Bauwerke diese Ganzheit real zu vermitteln scheinen. Wobei Ganzheit nicht nur räumlich, sondern melır noch zeitlich-historisch zu verstehen ist und die gesicherte Herkunft des Eigenen miteinschließt.

Der Ich-Erzähler in Blatters Roman formuliert diesen Zusammenhang zwischen der Sehnsucht nach Ganzheit und dem Erzählen folgendermaßen:

Die eigene Zeit und Welt der Sätze, manchmal ereifern wir uns darüber. Du erwartest von einem Buch, daß es ein Lebensbuch ist, dich ein wenig neu erfindet. Durch Wörter und Sätze hindurch willst du in einem anderen Buch lesen, im Buch deines Lebens. (...)

Ich habe Sicht auf den Nil, in jeden Satz, den ich schreibe, soll der Nil einfließen mit dem Geruch von Wasser, Schlamm, Fisch und Liebe. (W 328 f.)

Besser könnte man es kaum formulieren, wenn man die Funktion Ägyptens für das literarische Erzählen beschreiben will. Blatters IchErzähler entkommt in diesem zweigeteilten Roman „Wassermann" einem romantischen Ungenügen an der Normalität der Schweiz in ein Land, das er sich als eine mögliche Heimat zurechterzählt, in der er mythische Kräfte erleben wie verlorene Sinnlichkeit wiedergewinnen kann. Diese Sinnlichkeit, immer wieder in genauen Beschreibungen des Kairoer Stadtlebens wie des oberägyptischen Landlebens in Sprache geflochten, ist indessen eine von der - ägyptischen - Realität ganz abgelöste: die Fremde löst das sinnliche Erleben zwar aus, ist aber lediglich Ersatz und Stimulus für ein lange verlorenes Gefühl von Ganzheit, Heimat und Geborgenheit, das offensichtlich nur in der Fremde empfunden werden kann, wo die „wirkliche Wirklichkeit" nicht abzulenken vermag von der Selbst-Stilisierung des Ich. Folglich endet der Roman mit einer Heimatszene, die beide Erzählstränge des Romans und damit beide Orte die Schweiz und Ägypten - miteinander verknüpft, die Vergangenes und Gegenwärtiges vereint, und die in ihrer Stimmung aus Kellers Feder stammen könnte: 
Wenige Schritte entfernt, unter der Kôbri et-Tahrir, münden Reuss und Limmat in den Nil. Ein paar Kilometer stromaufwärts, auf gestampften Plätzen, füllen Freiämterbauern schneeweiße Baumwolle in große Säcke. In der Arbeitspause teilen sie mit den ägyptischen Bauern diese faden amerikanischen Kekse, grinsen einander zu, zeigen unverschämt schadhafte Zähne, verkuppeln Töchter und Söhne. Auf den Mutschellenpaß scheint die afrikanische Sonne, die Milane kreisen über dem Eichenwald, spüren, daß ihre Flügel Feuer fangen, stürzen mit panikartigen Schreien ab auf die Terrasse des grauen Hauses im Kreis 5 in Zürich. (W 389)

Die Kraft des Erzählens, seine Macht zur Welterschaffung, wird hier ebenso deutlich demonstriert wie das Illusionäre, das seine Grundbedingung ist. So wie die literarische Welt Gottfried Kellers dem heutigen Leser - wie auch dem Erzähler im „Wassermann“ - eine Welt sehnsüchtiger, rückwärtsgewandter Illusion ist, mit der Spuren der eigenen Herkunft und damit identitätsstiftende Elemente des eigenen Kulturraums verbunden sind, so enthält die Welt Ägyptens, wie sie in den beiden Romanen dargestellt ist, nicht minder illusionäre, aber ebenso an die Kultur Europas gebundene Identitätsmomente, die mit der gegenwärtigen ägyptischen Kultur und Gesellschaft wenig zu tun haben. Mehr noch: die literarische Darstellung der puren Gegenwärtigkeit Ägyptens ist kraft seiner die Illusion zerstörenden Anschaulichkeit in beiden Romanen das verfremdende Element, das Enttäuschung im positiven Sinne auslöst, indem es die illusionäre Täuschung bewußt macht und zu Erkenntnis führt. Eine Erkenntnis allerdings, der sich das literarische Erzählen immer wieder entgegenstellt, damit seine Möglichkeiten wie seine Grenzen aufzeigend. Der Schluß von Blatters Roman, in dem die Milane ,mit panikartigen Schreien [...] auf die Terrasse des grauen Hauses im Kreis 5 in Zürich" abstürzen, macht diese Grenzen deutlich, gleichzeitig damit das ästhetische Konzept des Romans, der ähnlich wie Werners Text allein dem Erzählen noch die Möglichkeit der Wiederherstellung kulturüberschreitender mentaler Konzepte in ein Ganzes zuschreibt.

In beiden Romanen dient das gegenwärtige Ägypten also nicht der Darstellung von Fremde, sondern vielmehr als Objekt verfremdender Darstellung eigener Konzepte von einem schon immer in (der eigenen) Sprache tradierten Kulturraum, den man logozentristisch als zum Eigenen gehörend vereinnahmt hat. Der Europäer kann nur das beschreiben, was 
er sieht, dabei vergessend, daß er es aus seiner Perspektive wahrnimmt, die nota bene alle anderen ausschließt. Ägypten in der Literatur bedeutet demnach die Auseinandersetzung mit diesen anderen Perspektiven, die die eigene nicht nur relativieren, sondern sie in ihrem alleingültigen Anspruch negieren. Die Funktion der Sprache dabei, besonders im Hinblick auf die Wirkungsweise ihrer literarischen Ästhetik, ist die Perspektivierung, d.h. die schichtende Zerlegung eines imaginierten Ganzen - hier: eines ganzheitlichen, aber relitätsfernen Ägyptenbildes.

Die Therapiesubstanz der Fremde schwindet im Moment der Begegnung mit ihr. Solange sie fern ist wie die vergangenen Zeiten, solange ihr in Archiven nachgespürt werden kann, hält sich der in der eigenen Gegenwart verlorene Europäer im eigenen Erklärungsraum auf, der zugleich identitätsstiftender Spielraum ist. In diesem Erklärungs- und Spielraum erzählt sich Ägypten - bei Werner wie bei Blatter. Die Suche nach dem bewunderten Großvater in Werners Roman, die Verknüpfung seiner Biographie mit dem Land Ägypten, in dem er lange gelebt hatte, bzw. das Wiederentdecken der Kellerschen Welt in Oberägypten, lassen die zeitliche wie die räumliche Distanz in der Erzählung als gleich und gleichermaßen überwindbar aufscheinen. Die Geborgenheit im Archiv wie das Aufgehobensein im Erzählen schwinden jedoch jäh bei der Rückkehr in die gegenwärtige, eigene Welt, die keine Rückkehr „ins Vertraute" ist, wie die weiter oben zitierte Textstelle deutlich werden ließ.

Ägypten in der Literatur ist nicht Ägypten und ist es doch. Verblüffend genaue Beschreibungen von Orten, Ereignissen und des Lebens in Ägypten sind kunstvoll vermittelte Wahrnehmungsspiegelungen, die zwar ausschnittweise das Land, mehr noch aber den Wahrnehmenden beschreiben. Warum Ägypten, die Frage nach dem gewählten Land, ist nichl dic relevante Frape - z.n Ägypten pilb es keine Altermative. Dic eigentliche Frage lautet eher: Warum Ägypten, und zielt damil aul dic Befindlichkeiten des europäischen Selbst. 


\section{Zusammenfassung}

\section{Die Funktion von Bildern und Motiven zu Ägypten und Kairo in der} deutschsprachigen Literatur der Gegenwart

Die Funktion von Bildern und Motiven zu Ägypten und Kairo in der delitschsprachigen Literatur der Gegenwart:Welche Funktion hat die Beschreibung eines fremden Landes, einer fremden Stadt in einem literarischen Text deutscher Sprache? Zielt die Beschreibung auf die literarische Darstellung einer geographischen verortbaren Größe, oder geht es in der Literatur nicht immer um anderes als den fremden Ort, der lediglich Raum für die Erfahrungen des erzählten Subjektes ist? Meist wird dem Ort in der Fremde - bei aller detailgetreuen Referenz - eine Bestimmung zugewiesen, die nur sehr wenig mit der zu erlebenden Realität $\mathrm{zu}$ tun hat. Eher bezieht sich Literatur auf eigene Rezeptionsweisen dieses fremden Ortes, die Mythos und Historie, Gegenwärtige Vorurteile und stereotype Bilder zu einer Substanz verdichten, die dem Autor als Ausgangsmaterial für seine literarischen Verfremdungen dienen. Dabei dient der Prozess der literarischen Gestaltung dieses Materials der Enttäuschung:

sowohl der eigene wie der fremd Ort taugen nur bedingt für den Entwurf einer eigenen Geschichte, die nie als Ganzes zu beschreiben ist.

Anhand mehrerer Texte, die in den letzten 20 Jahren im deutschsprachigen Raum entstanden sind und Ägypten und Kairo zum Schauplatz machen, soll den oben skizzierten Fragen und Thesen nachgegangen werden. 


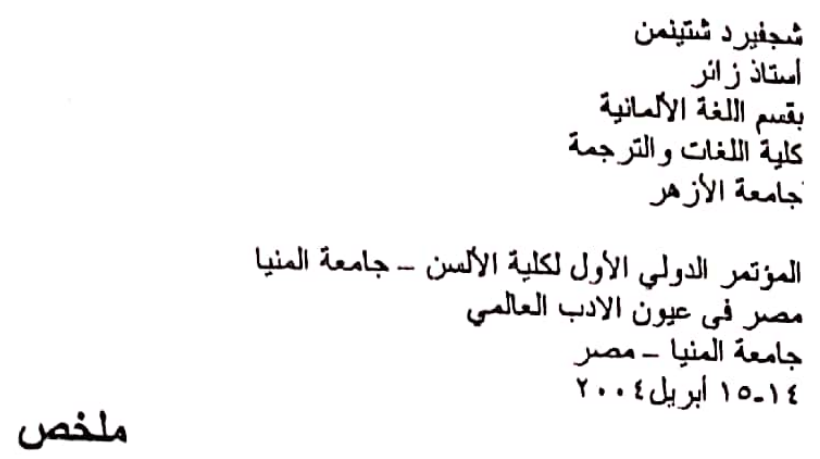

\section{وظيفة الصسود و العناصر المتعلقة بمصر والقاهرة}

فى الأدب المعاصر للدول الناطقة بالألمانية

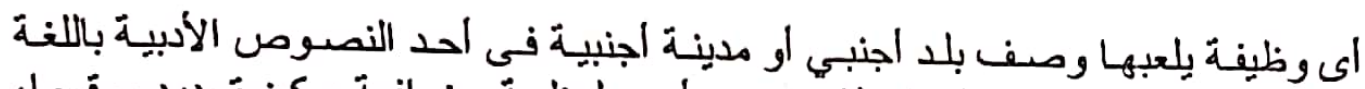

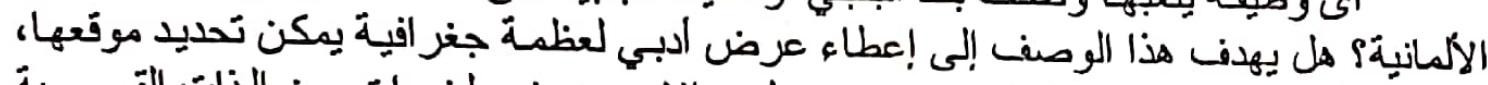

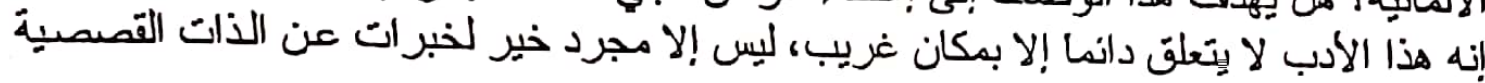
أو المحكية؟

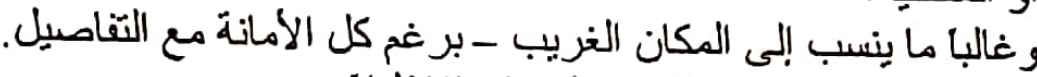

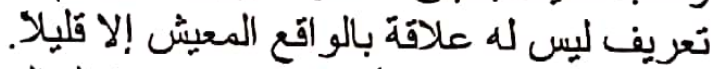

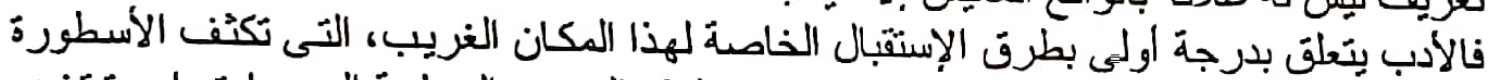

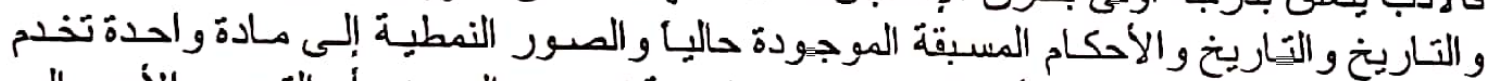

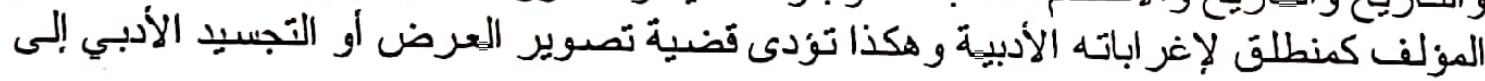

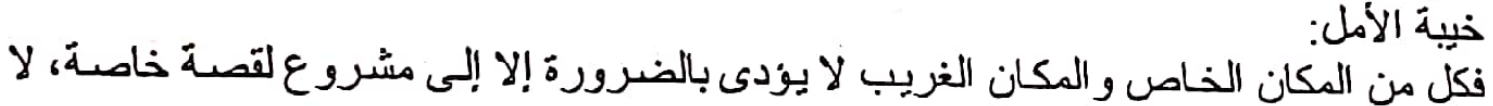
يمكن وصفها ككل منكامل.

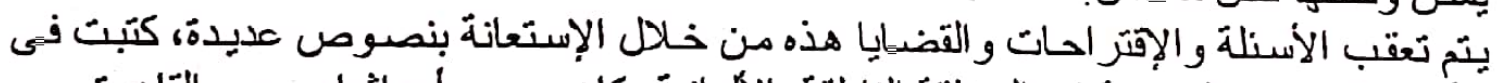
الأعو ام العشرين الماضية فى المنطقة الناطقة بالألمانية، كان مسر ح الحداثها مصر و القاهرة. 